\title{
Teor de flavonóides totais em produtos contendo pata-de-vaca (Bauhinia L.) comercializados em farmácias de Recife/PE
}

PEIXOTO SOBRINHO, T.J.S. ${ }^{1 *}$; GOMES, T.L.B. ${ }^{1}$; CARDOSO, K.C.M. ${ }^{1}$; ALBUQUERQUE, U.P. ${ }^{2}$; AMORIM, E.L.C. ${ }^{1}$ ${ }^{1}$ Laboratório de Produtos Naturais, Departamento de Ciências Farmacêuticas, Centro de Ciências da Saúde, Universidade Federal de Pernambuco, Avenida Prof. Arthur de Sá, CEP: 50670-901, Recife-Brasil *tadeu1903@yahoo.com.br ² Laboratório de Etnobotânica Aplicada, Área de Botânica, Departamento de Biologia, Universidade Federal Rural de Pernambuco, Rua Dom Manoel de Medeiros, CEP: 52171-900, Recife-Brasil

\begin{abstract}
RESUMO: O objetivo deste estudo foi avaliar, através de metodologia espetrofotométrica, o teor de flavonóides totais em produtos contendo pata-de-vaca (Bauhinia L.) comercializados em farmácias de Recife, estado de Pernambuco, Brasil. Foi utilizada metodologia de complexação com cloreto de alumínio. Os resultados foram expressos em $\mu \mathrm{g}$ equivalentes de rutina por mililitro

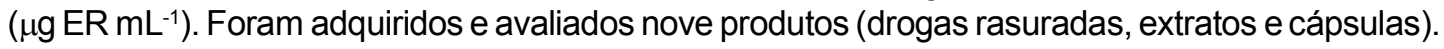
As concentrações de flavonóides totais das amostras variaram de 1,663 $\pm 0,144$ a 28,698 \pm $0,187 \mu \mathrm{g} \mathrm{ER} \mathrm{mL}^{-1}$. Os resultados não apresentaram uniformidade quanto à concentração de flavonóides totais, indicando falta de padronização nas preparações fitoterápicas e possível ineficácia dos produtos comercializados.
\end{abstract}

Palavras-chave: pata-de-vaca, padronização, espectrofotometria

\begin{abstract}
Total flavonoid content in products containing "pata-de-vaca" (Bauhinia L.) sold in pharmacies in Recife/PE. The aim of this study was to evaluate, through spectrophotometric methodology, the total flavonoid content in products containing "pata-de-vaca" (Bauhinia L.) sold in pharmacies in Recife, state of Pernambuco, Brazil. The methodology of complexation with aluminum chloride was used. The results were expressed as $\mu \mathrm{g}$ of rutin equivalents per milliliter ( $\left.\mu \mathrm{g} \mathrm{RE} \mathrm{mL} \mathrm{m}^{-1}\right)$. Nine products (drug erasures, extracts and capsules) were purchased and evaluated. The concentrations of total flavonoids in the samples ranged from $1.663 \pm 0.144$ to $28.698 \pm 0.187 \mu \mathrm{g} \mathrm{RE} \mathrm{mL}-1$. The results showed no uniformity as to the total flavonoid content, indicating a lack of standardization in herbal preparations and a possible inefficiency of the marketed products.
\end{abstract}

Key words: "pata-de-vaca", standardization, spectrophotometry

\section{INTRODUÇÃO}

O gênero Bauhinia L. (Caesalpiniacaea) possui aproximadamente 300 espécies, sendo que no Brasil ocorrem cerca de $20 \%$ destas (Haver, 2002), onde são utilizadas na medicina tradicional, vendidas em feiras livres ou encontradas na composição de diversos fitoterápicos (Melo et al., 2004). Estas espécies apresentam diversas indicações populares, principalmente como tônico, expectorante, hipocolesterolemiante, hipoglicemiante, antihipertensiva e antiinflamatório (Silva \& Cechinel Filho, 2002; Almeida et al., 2006; Albuquerque, 2006; Albuquerque et al., 2007; Agra et al., 2007). Nos fitoterápicos à base da pata-de-vaca (gênero Bauhinia L.), ainda que não seja preconizada nos códigos oficiais, considera-se a folha como a parte vegetal a ser utilizada para fins terapêuticos, uma vez que é o órgão utilizado na medicina popular.

Estudos apontam que os flavonóides são os marcadores químicos e responsáveis pelas diversas atividades farmacológicas desempenhadas pelo gênero Bauhinia (Silva et al., 2002; Argolo et al., 2004; Sousa et al., 2004). Almeida et al. (2006) avaliaram a atividade antidiabética do extrato foliar de Bauhinia cheilandra (Bong.) Steudel sobre os níveis de glicose em ratos diabéticos induzido por aloxana e encontraram que os tratamentos nas doses de 300, 600 e $900 \mathrm{mg} \mathrm{kg}^{-1}$, apresentaram atividade hipoglicemiante significativa. Em outro estudo com diferentes frações do extrato foliar de Bauhinia candicans Benth, os resultados mostraram que o tratamento utilizando doses de 8 $\mathrm{mg} \mathrm{kg}^{-1}$ da fração III (kaempferol-3-O- $\beta$-rutinosídeo)

Recebido para publicação em 09/07/2010

Aceito para publicação em 25/04/2012

Rev. Bras. PI. Med., Botucatu, v.14, n.4, p.586-591, 2012. 
reduziu significativamente os níveis de glicemia de coelhos com diabetes induzida por aloxano, evidenciando uma potente atividade hipoglicemiante do flavonóide (Fuentes et al., 2004).

Mesmo sendo o composto majoritário no vegetal, diversos fatores podem modificar a rota metabólica dos flavonóides e, com isto, alterar a composição dos insumos para a produção de medicamentos. Alterações ambientais como sazonalidade, ritmo circadiano, radiação, temperatura, altitude e umidade, interferem qualitativamente e/ou quantitativamente no teor dos princípios-ativos presentes no vegetal (Gobbo-Neto \& Lopes, 2007). Fatores laboratoriais como estabilização, sistema extrativo, tempo e temperatura de armazenamento também podem influenciar o teor dos metabólitos ativos e alterar a constância e eficácia do produto acabado (Falkenberg et al., 2004; Andreo \& Jorge, 2006).

Diversas técnicas podem ser empregadas para a detecção e doseamento de flavonóides em amostras vegetais, tais como a Cromatografia Líquida de Alta Eficiência (CLAE), Cromatografia Gasosa (CG), Cromatografia Líquida associada à Espectrometria de Massas (CL-EM), Cromatografia em Camada Delgada (CCD), entre outras. No entanto, o método de quantificação de flavonóides por espectrofotometria, em relação às demais, é uma técnica mais acessível, prática e menos onerosa e, devido às duplas ligações presentes nos anéis aromáticos, os flavonóides podem ser analisados na região do ultravioleta ou visível (Markhan, 1982).

Na reação, o íon alumínio $\left(\mathrm{Al}^{3+}\right)$ complexase com as moléculas de flavonóides da amostra, estabelecendo o complexo estável flavonóide-A ${ }^{3+}$ de coloração amarela cuja intensidade é proporcional à concentração de flavonóide presente na amostra (Mabry et al., 1970). Esta complexação promove, em espectroscopia, deslocamento batocrômico e intensificação das absorções, podendo ser quantificado sem sofrer influência de outros compostos fenólicos presentes na amostra (Figura 1).

De acordo com a Agência Nacional de Vigilância Sanitária (ANVISA) fitoterápicos são medicamentos obtidos a partir de plantas medicinais, empregando-se exclusivamente derivados de droga vegetal e assim como todo medicamento, deve oferecer garantia de qualidade, ter efeitos terapêuticos comprovados, composição padronizada e segurança

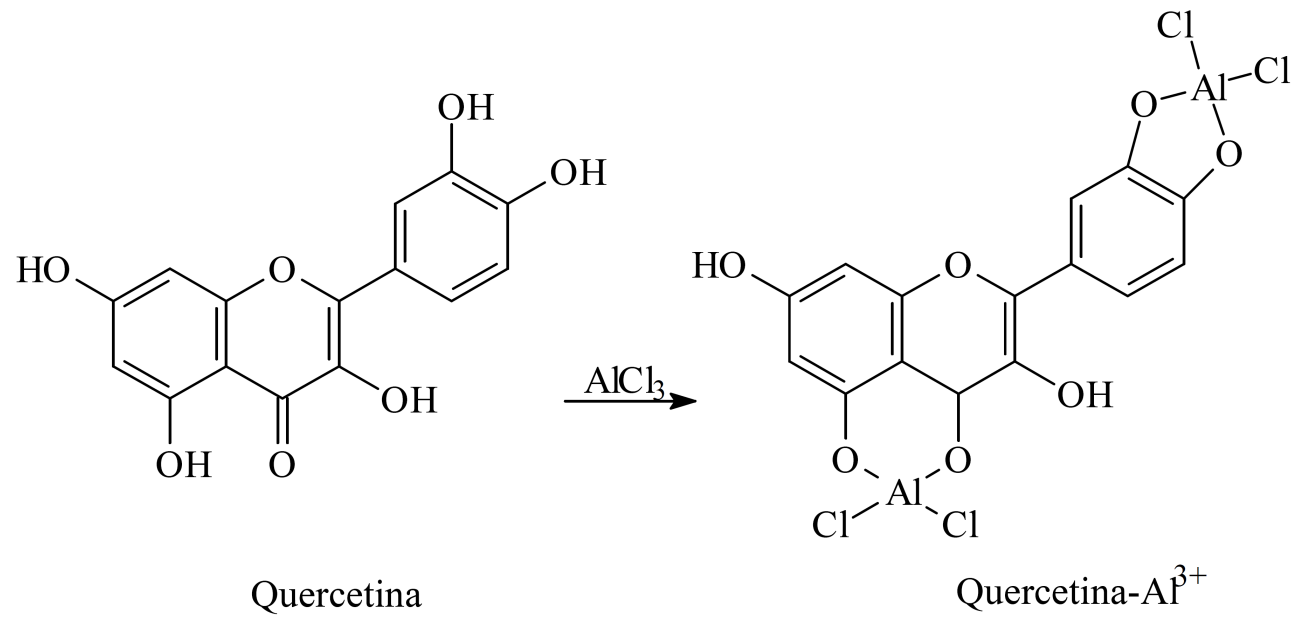

FIGURA 1. Esquema ilustrado da complexação do flavonóide quercetina com cloreto de alumínio $\left(\mathrm{AlCl}_{3}\right)$ o qual forma o complexo estável flavonóide-Al ${ }^{3+}$ (Mabry et al. 1970).

de uso para a população (Brasil, 2009). A qualidade deve ser alcançada mediante o controle de qualidade das matérias-primas, do produto acabado, materiais de embalagem, formulação farmacêutica e estudos de estabilidade (Brasil, 2009).

Com base na importância científica e econômica que as espécies de Bauhinia despertam, o objetivo deste trabalho foi avaliar, por espectrofotometria, o conteúdo de flavonóides totais de produtos fitoterápicos contendo plantas do gênero Bauhinia comercializados em farmácias de Recife, estado de Pernambuco.

\section{MATERIAL E MÉTODO}

\section{Equipamentos}

Foi utilizado no estudo agitador eletromagnético e tamises Bertel, balança analítica eletrônica ShimadzuAX200, cubeta de vidro Equilab de $10 \mathrm{~mm}$ de caminho óptico, espectrofotômetro Shimadzu UV-Mini 1240 e placa de aquecimento Tecnal TE-018.

\section{Reagentes e padrão de referência}

As análises quantitativas foram realizadas com os seguintes solventes: metanol p.a. (Vetec), 
ácido acético glacial p.a. (Merck), solução metanólica de piridina a $20 \%$ (Vetec) e reagente cloreto de alumínio em metanol a $50,0 \mathrm{mg} \mathrm{mL}^{-1}$ (Vetec). Como padrão para flavonóides foi empregado rutina 99,5\% (Merck).

\section{Metodologia analítica}

Seguiu-se a metodologia descrita por Peixoto Sobrinho et al. $(2008,2009,2010)$. Os extratos foram preparados com $500,0 \mathrm{mg}$ das amostras pulverizadas com granulometria de 60 Mesh em erlenmeyers de $50 \mathrm{~mL}$, aos quais foram adicionados $25,0 \mathrm{~mL}$ de metanol $80 \%$ e extraídos, em placa de aquecimento durante 30 minutos, sendo filtrados para balões volumétricos de $50 \mathrm{~mL}$. Em cada erlenmeyer foi adicionado $20,0 \mathrm{~mL}$ do solvente e novamente filtrado, aferindo-se o volume do balão com metanol $80 \%$. Deste extrato foi pipetada e transferida alíquota de $1,0 \mathrm{~mL}$ para balão volumétrico de $25 \mathrm{~mL}$, ao qual foram acrescentados $0,6 \mathrm{~mL}$ de ácido acético glacial, $10,0 \mathrm{~mL}$ da solução metanólica de piridina e $2,5 \mathrm{~mL}$ do reagente cloreto de alumínio, completando-se o volume com água destilada. Após 30 minutos em temperatura ambiente e ao abrigo da luz, as leituras foram realizadas em espectrofotômetro a $420 \mathrm{~nm}$ em cubetas de vidro. As amostras foram analisadas utilizando-se cinco réplicas para cada produto e os resultados foram expressos em $\mu \mathrm{g}$ equivalentes de rutina por mililitro $\left(\mu \mathrm{gER} \mathrm{mL}^{-1}\right)$.

Para construção das curvas de calibração, foram preparadas soluções metanólicas de rutina a $0,5 \mathrm{mg} \mathrm{mL}^{-1}$, do qual foram retiradas sete alíquotas para balões volumétricos de $25 \mathrm{~mL}$. Em cada balão foi acrescentado $0,6 \mathrm{~mL}$ de ácido acético glacial, 10,0 $\mathrm{mL}$ da solução de piridina e $2,5 \mathrm{~mL}$ do reagente cloreto de alumínio, completando-se o volume com água destilada, obtendo-se concentrações finais de 1,0$40,0 \mu \mathrm{g} \mathrm{ER} \mathrm{mL} \mathrm{mL}^{-1}$. Após 30 minutos em temperatura ambiente e ao abrigo da luz, as leituras foram realizadas em espectrofotômetro a $420 \mathrm{~nm}$ em cubetas de vidro, utilizando-se água destilada como solução-branco.

\section{Controle de qualidade dos produtos}

Seis produtos contendo plantas do gênero Bauhinia, sendo três na forma de droga rasurada (Grupo I: Marcas A, B e C) e três na forma de cápsula (Grupo II: Marcas D, E e F) e três fitoterápicos na forma de extrato (Grupo III: Marcas G, H e I), foram adquiridos no mês de maio/2008 na cidade do Recife, estado de Pernambuco.

A quantificação do conteúdo flavonônico das amostras na forma de droga rasurada e cápsula seguiu conforme o descrito na metodologia analítica. A análise das formas farmacêuticas líquidas procedeu diretamente no extrato, sendo retiradas alíquotas de 1,0 mL e transferidas para balões volumétricos de $25 \mathrm{~mL}$, onde se procedeu a adição dos reagentes utilizados para a quantificação. Os resultados foram expressos como $\mu \mathrm{g}$ equivalente de rutina por mililitro.

\section{Análise estatística}

Foi utilizado o teste de Kolmogorov-Smirnov para avaliar a normalidade dos resultados e análise de variância ANOVA um critério para comparar os teores de flavonóides totais no grupo formado pela mesma forma farmacêutica, complementando-se o estudo com o teste de Tukey. As análises foram realizadas ao nível de $95 \%$ de confiança $(\alpha=0,05)$ através do programa BioEstat 5.0 (Ayres et al., 2007).

\section{RESULTADO E DISCUSSÃO}

A equação de correlação e o coeficiente de determinação na construção da curva de calibração utilizada para analisar os teores de flavonóides totais dos produtos foram, respectivamente, $y=0,0262 x+$ 0,0007 e $R^{2}=0,9998$ (Figura 2).

$\mathrm{Na}$ análise dos produtos a base de plantas do gênero Bauhinia, foi observada diferenças estatísticas dentro dos grupos formados pela mesma forma farmacêutica ( $<<0,0001)$. Os resultados das análises de controle de qualidade dos produtos são mostrados na Tabela 1 e Figura 3.

A amostra $G$ apresentou a maior concentração de flavonóides totais $(28,698 \pm 0,187$ $\left.\mu \mathrm{g} \mathrm{ER} \mathrm{mL}^{-1}\right)$, seguida pela amostra $\mathrm{A}(26,511 \pm 0,291$

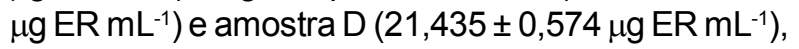
enquanto os menores níveis foram observados paras

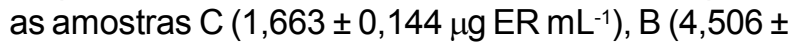
$0,714 \mu \mathrm{g}$ ER $\left.\mathrm{mL}^{-1}\right)$ e $\mathrm{F}\left(6,454 \pm 0,225 \mu \mathrm{g}\right.$ ER $\left.\mathrm{mL}^{-1}\right)$. Comparando as médias das concentrações de flavonóides totais de mesma forma farmacêutica, os extratos apresentaram um coeficiente de variação (CV = $41,26 \%$ ), seguido por cápsulas (CV $=63,12 \%$ ) e, por último, as amostras na forma de droga rasurada, que apresentaram alta variação (CV $=124,85 \%)$. Isto mostra que não há padronização quanto aos níveis de flavonóides totais presentes nos produtos comercializados, sendo a droga rasurada a forma que apresentou heterogeneidade mais expressiva.

A análise quantitativa de matérias-primas vegetais e/ou fitoterápicos torna-se um processo fundamental para o controle de qualidade e, isto conduz à segurança, estabilidade, constância e eficácia dos fitomedicamentos produzidos (Bara et al., 2004).

Oliveira \& Beretta (2007) constataram variação entre os teores de flavonóides totais contidos em insumos farmacêuticos de Calendula officinalis L. (Asteraceae) usadas em farmácias magistrais, principalmente na comparação entre tinturas e os respectivos extratos glicólicos, de forma que os extratos apresentaram teores correspondentes a 10\% 


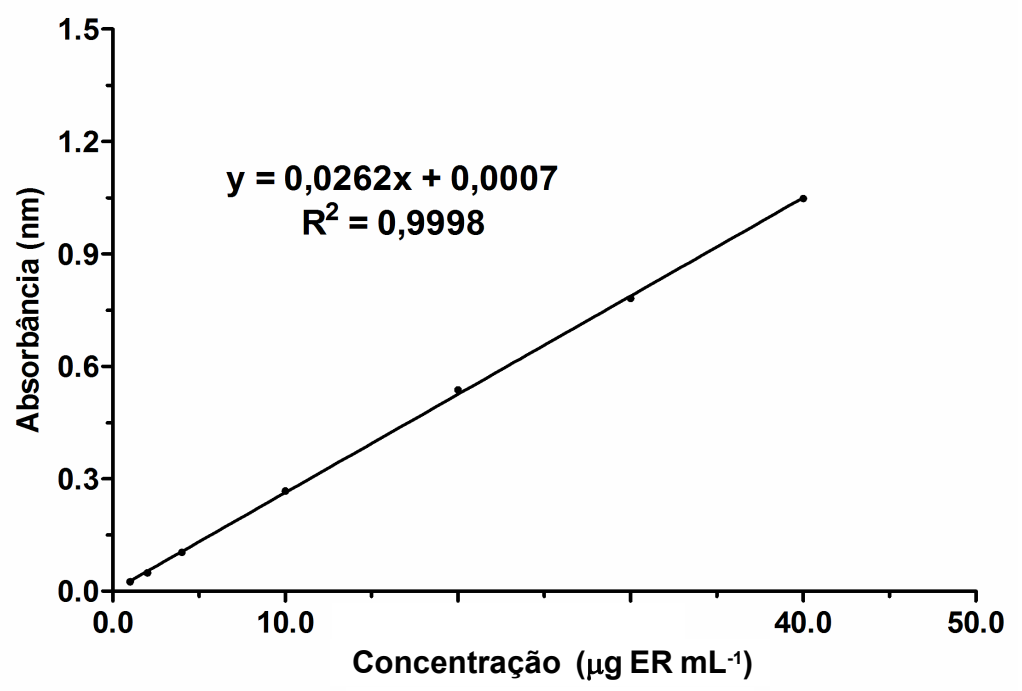

FIGURA 2. Curva de calibração construída com rutina nas concentrações de 1-40 $\mu \mathrm{g} \mathrm{mL}^{-1}$ a $420 \mathrm{~nm}$, onde a equação de correlação e o coeficiente de determinação obtidos foram y =0,262x +0,0007 e $R^{2}=0,9998$.

TABELA 1. Determinação das concentrações de flavonóides totais em $\mu \mathrm{g}$ equivalentes de rutina por mililitro ( $\mu \mathrm{g}$ ER $\mathrm{mL}^{-1}$ ) contidos em nove produtos a base de plantas do gênero Bauhinia comercializados em farmácias do Recife/PE.

\begin{tabular}{ccccc}
\hline $\begin{array}{c}\text { Forma } \\
\text { farmacêutica }\end{array}$ & Marca & $\begin{array}{c}\text { Descrição } \\
\text { taxonômica * }\end{array}$ & $\begin{array}{c}\text { Concentração } \pm \text { DP } \\
\left.(\mu \mathrm{g} \mathrm{ER} \mathrm{mL})^{-1}\right)\end{array}$ & CV $(\%)$ \\
\hline Droga rasurada & A & Bauhinia spp. & $26,511 \pm 0,291$ & 1,10 \\
& B & Bauhinia sp. & $4,506 \pm 0,714$ & 15,84 \\
& C & Bauhinia forficata Link & $1,663 \pm 0,144$ & 8,67 \\
\hline Cápsula & D & Bauhinia sp. & $21,435 \pm 0,574$ & 2,68 \\
& E & Bauhinia sp. & $9,629 \pm 0,103$ & 1,07 \\
& F & Bauhinia sp & $6,454 \pm 0,225$ & 3,49 \\
\hline & G & Bauhinia forficata & $28,698 \pm 0,187$ & 0,65 \\
& H & Bauhinia sp & $19,838 \pm 0,137$ & 0,69 \\
& I & Bauhinia forficata L.K. & $12,044 \pm 0,253$ & 2,10 \\
\hline
\end{tabular}

$\mathrm{DP}=$ Desvio-Pe $\quad$ io; $\mathrm{CV}=$ Coeficiente de Variação; *identificação que consta no produto.

da melhor tintura, demonstrando a necessidade da determinação de especificações técnicas que garantam qualidade do produto final. Estudo realizado com matérias-primas e cápsulas contendo Ginkgo biloba L. (Ginkgoaceae), mostrou que 33\% e 91\%, respectivamente, dos teores de flavonóides estavam em desacordo com as especificações declaradas pelos fabricantes (Bara et al., 2004).

A utilização de insumos farmacêuticos com teores de princípios-ativos abaixo das especificações leva a produção de fitoterápicos sem uniformidade química, podendo reduzir a eficiência dos fitomedicamentos. Portanto, é importante enfatizar a necessidade de realização de análises para determinação do teor de princípios ativos em matérias- primas vegetais, associadas às análises de identidade das plantas. Desta forma pode-se buscar uma padronização de fitoterápicos e consequentemente, contribuir para assegurar eficácia do uso.

Concluímos que os produtos fitoterápicos contendo plantas do gênero Bauhinia L. que são amplamente vendidos nas farmácias da cidade do Recife/PE não apresentam uniformidade quanto ao conteúdo de flavonóides totais. Considerando que a ação hipoglicemiante desempenhada por extratos de diversas espécies vegetais é atribuída a flavonóides, a simples constatação de diferenças significativas no teor desse grupo de substâncias fenólicas nas várias amostras analisadas permite inferir que esses produtos vegetais comercializados em Recife não 


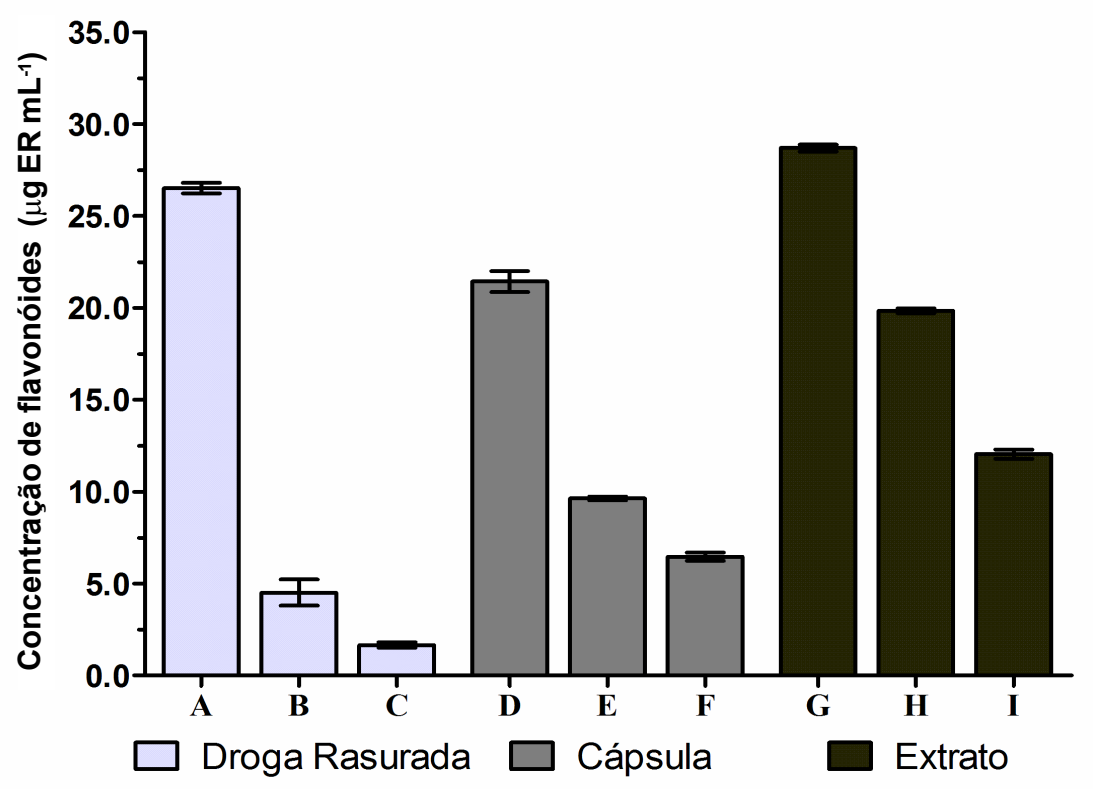

FIGURA 3. Concentrações de flavonóides totais em $\mu \mathrm{g}$ equivalentes de rutina por mililitro $\left(\mu \mathrm{g} \mathrm{ER} \mathrm{mL}^{-1}\right)$ contidos em produtos a base de plantas do gênero Bauhinia L. comercializados em farmácias do Recife/PE.

possuem homogeneidade terapêutica. Além disto, muitos dos produtos não continham a designação da espécie, podendo ocorrer à adulteração com outras espécies vegetais.

\section{AGRADECIMENTO}

Os autores à Coordenação de Aperfeiçoamento de Pessoal do Ensino Superior (CAPES) pela concessão de Bolsa de Mestrado à T.J.S. Peixoto Sobrinho, a FACEPE/CNPq pela Bolsa de Iniciação Científica concedida a K.C.M. Cardoso, a PROPESQ pela Bolsa de Iniciação Científica concedida a T.L.B. Gomes e ao CNPq pelo suporte financeiro concedido através do "Edital Universal" a E.L.C. Amorim.

\section{REFERÊNCIA}

AGRA, M.F. et al. Synopsis of the plants known as medicinal and poisonous in Northeast of Brazil. Revista Brasileira Farmacognosia, v.17, n.1, p.114-40, 2007. ALBUQUERQUE, U.P. Re-examining hypotheses concerning the use and knowledge of medicinal plants: a study in the Caatinga vegetation of NE Brazil. Journal of Ethnobiology and Ethnomedicine, v.2, n.30, p.1-10, 2006. ALBUQUERQUE, U.P. et al. Medicinal plants of the Caatinga (semi-arid) vegetation of NE Brazil: a quantitative approach. Journal of Ethnopharmacology, v.114, p.325-54, 2007.

ALMEIDA, E.R. et al. Hypoglycemic effect of Bauhinia cheilandra in rats. Fitoterapia, v.77, p.276-8, 2006.

ANDREO, D.; JORGE, N. Antioxidantes naturais: técnicas de extração. Boletim do Centro de Pesquisa de Processamento de Alimentos, v.24, n.2, p.319-36, 2006. ARGOLO, A.C.C. et al. Antioxidant activity of leaf extracts from Bauhinia monandra. Bioresource Technology, v.95, p.229-33, 2004.

AYRES, M. et al. BioEstat 5.0: aplicações estatísticas nas áreas das ciências biomédicas. Belém: Instituto de Desenvolvimento Sustentável Mamirauá, 2007. 339p. BARA, M.T.F. et al. Determinação de ginkgoflavonóides por cromatografia líquida de alta eficiência em matériasprimas e produtos acabados. Revista Eletrônica de Farmácia, v.1, n.1, p.1-7, 2004.

BRASIL, Agência Nacional de Vigilância Sanitária (ANVISA). Disponível em: <http://www.anvisa.gov.br>. Acesso em: 20 out. 2009.

FALKENBERG, M.B. et. al. Introdução à análise fitoquímica. In: SIMÕES, C.M.O. et al. (Eds.) Farmacognosia: da planta ao medicamento. 5.ed. Porto Alegre/Florianópolis: Editora da UFRGS/UFSC, 2004, p.229-46.

FUENTES, O. et. al. Hypoglycemic activity of Bauhinia candicans in diabetic induced rabbits. Fitoterapia, v.75, p.527-32, 2004.

GOBBO-NETO, L.; LOPES, N.P. Plantas medicinais: fatores de influência no conteúdo de metabólitos secundários. Química Nova, v.30, n.2, p.374-81, 2007. HAVER, N.J. Desenvolvimento, purificação e caracterização de lgG anti-lectina de folha de Bauhinia monandra. 2002. 95p. Tese (Doutorado - Ciências Biológicas) - Centro de Ciências Biológicas, Universidade Federal de Pernambuco, Recife.

MABRY, T.J. et. al. The systematic identification of flavonoids. Springer: Berlin, 1970. p.51-2.

MARKHAN, K.R. Techniques of flavonoid identification. Academic Press: London, 1982, 113p.

MELO, J.G. et al. Avaliação da qualidade de amostras comerciais de boldo (Peumus boldus Molina), pata-de- 
vaca (Bauhinia spp.) e ginco (Ginkgo biloba L.). Revista Brasileira de Farmacognosia, v.14, n.2, p.111-20, 2004. OLIVEIRA, A.H.; BERETTA, A.A. Avaliação da qualidade de insumos farmacêuticos a base de calêndula e própolis utilizados pelas farmácias magistrais. Revista Eletrônica de Farmácia, v.4, n.2, p.169-74, 2007.

PEIXOTO SOBRINHO, T.J.S. et al. Validação de metodologia espectrofotométrica para quantificação dos flavonóides de Bauhinia cheilantha (Bongard) Steudel. Revista Brasileira de Ciências Farmacêuticas, v.44, n.4, p.683-9, 2008.

PEIXOTO SOBRINHO, T.J.S. et al. Análise da pluviosidade e do efeito de borda sobre os teores de flavonóides em Bauhinia cheilantha (Bong.) Steud., Fabaceae. Revista Brasileira de Farmacognosia, v.19, p.740-5, 2009.
PEIXOTO SOBRINHO, T.J.S. et al. Otimização de metodologia analítica para o doseamento de flavonóides de Bauhinia cheilantha (Bongard) Steudel. Química Nova, v.33, p.288-91, 2010.

SILVA, R.L.; CECHINEL FILHO, V. Plantas do gênero Bauhinia: composição química e potencial farmacológico. Química Nova, v.25, n.3, p.449-54, 2002.

SILVA, F.R.M.B. et al. Acute effect of Bauhinia forficata on serum glucose levels in normal and alloxan-induced diabetic rats. Journal of Ethnopharmacology, v.83, p.337, 2002.

SOUSA, E. et al. Hypoglycemic effect and antioxidant potential of kaempferol-3,7-O-(á)-dirhammnoside from Bauhinia forficata leaves. Journal of the Natural Products, v.67, p.829-32, 2004. 